\title{
دور سيادة القانون في بناء السلام \\ (دراسة في محركات الصراع والأداء ألواء المؤسسي)
}

Doi: 10.23918/ilic2021.31

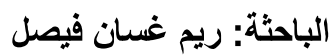

دبلوم عالي في دراسات بناء السلام/ جامعة الموصل

Reemghassan1313@gmail.com

\section{المقدمة}

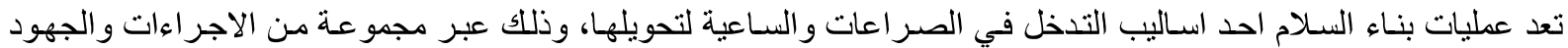

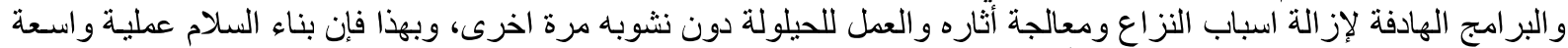

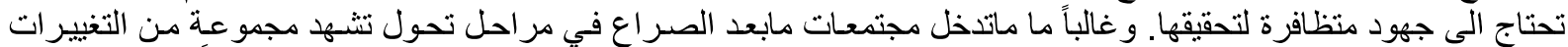

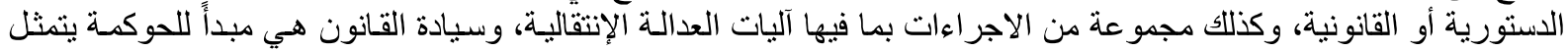

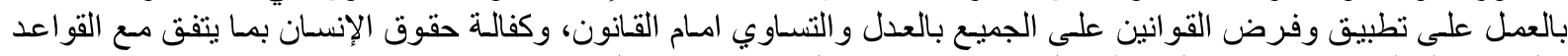

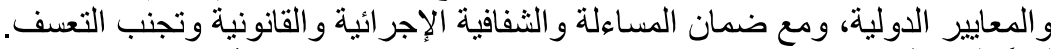

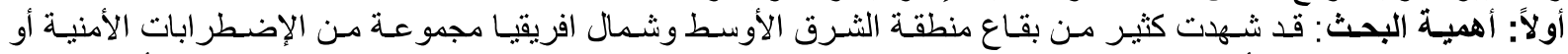

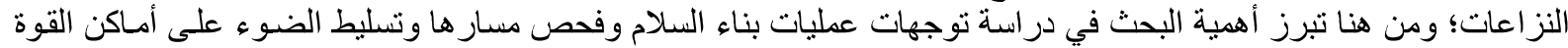

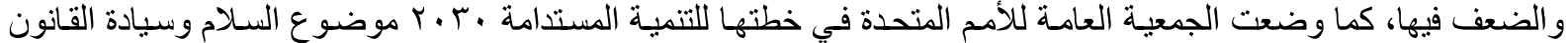

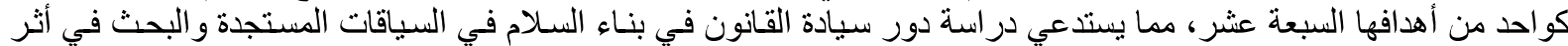

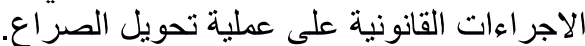

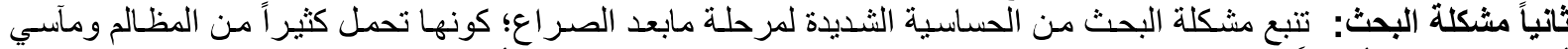

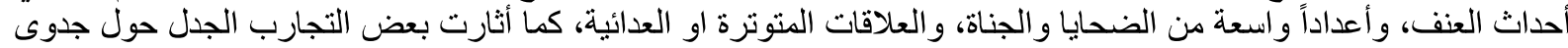

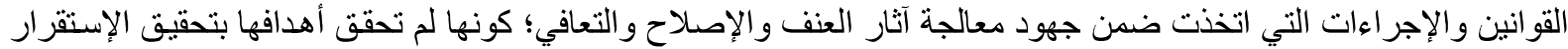

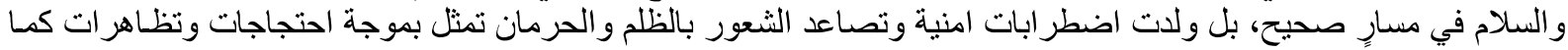

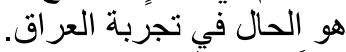

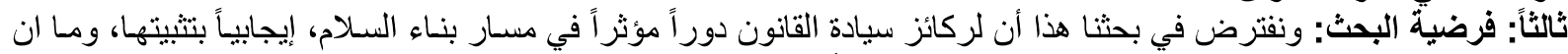

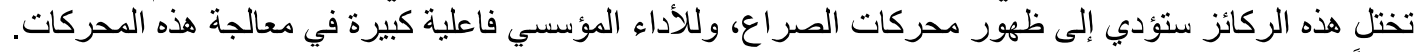

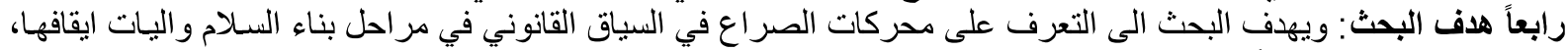

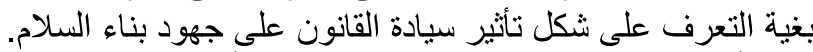

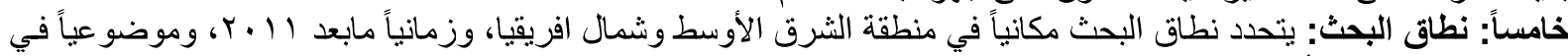

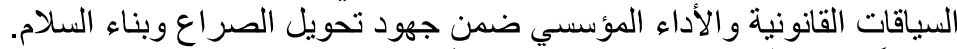

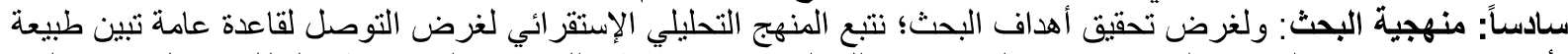

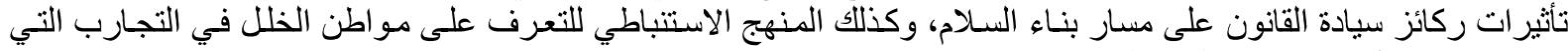

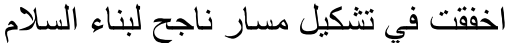

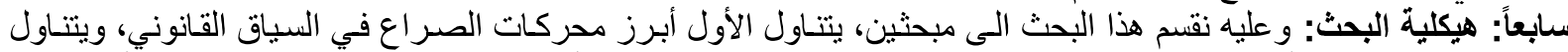

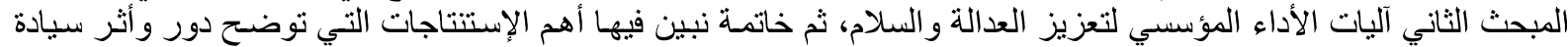
القانون في عمليات بناء السلام.

\section{المبحث الأول}

\section{محركات الصراع في السياقات القانول المباتونية}

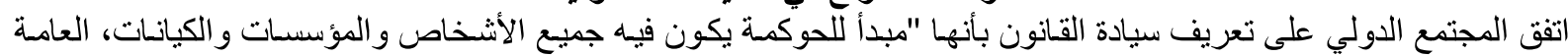

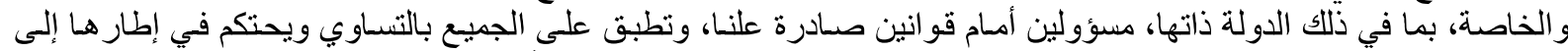

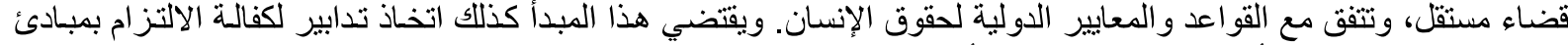

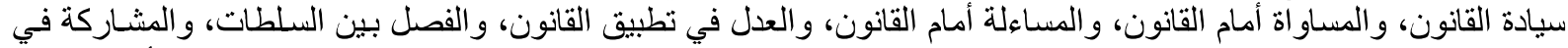

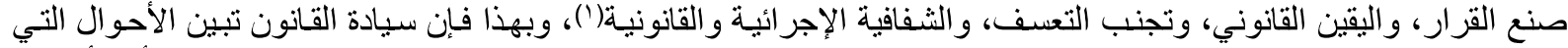

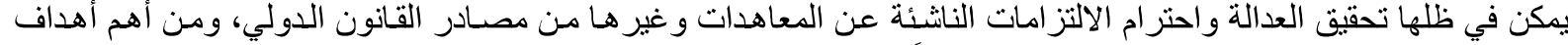

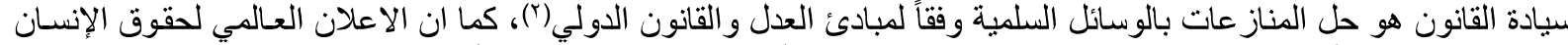

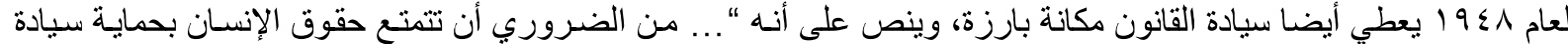

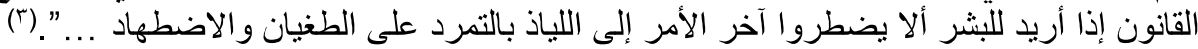

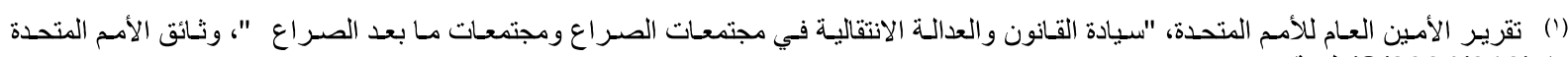

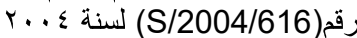

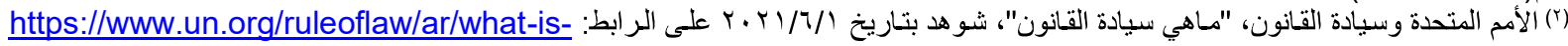
the-rule-of-law

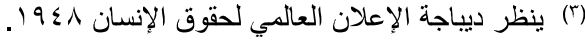




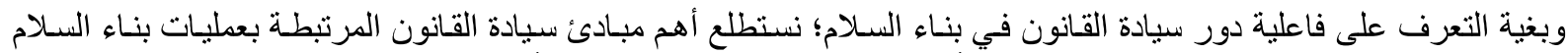

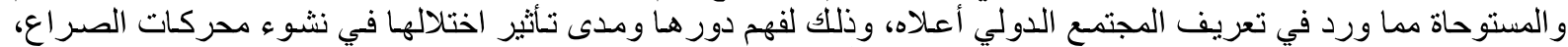

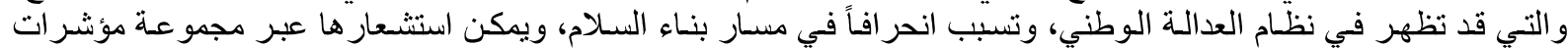

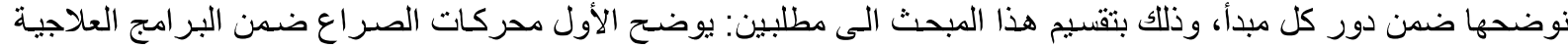

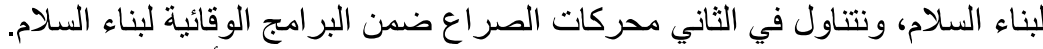

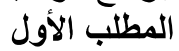

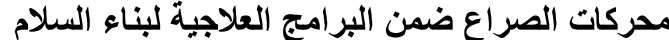

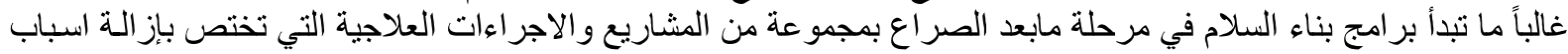

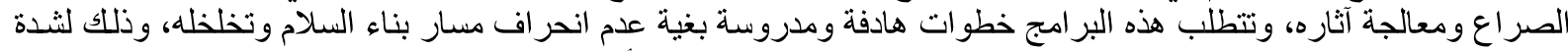

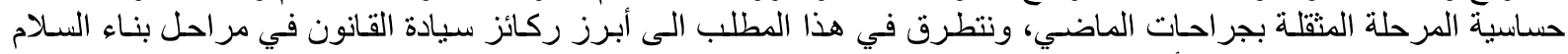
ودور ها في التوجيه نحو السلام أو الصراع. الصنافي القرع الأول

المساءلة وعدم الإفلات من العقاب

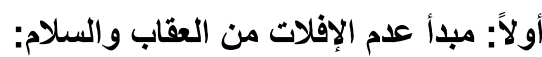

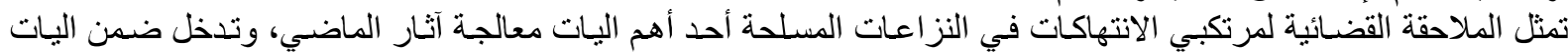

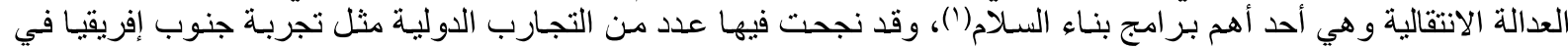

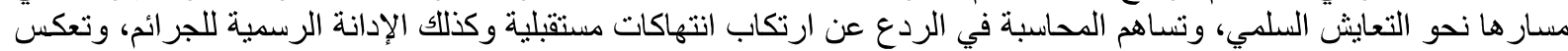

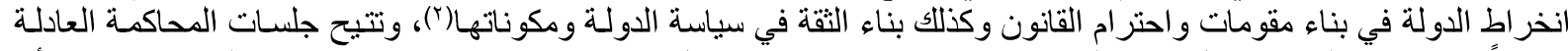

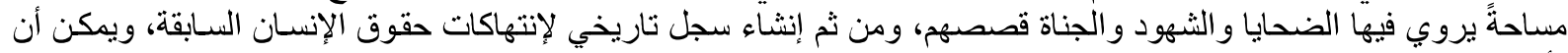

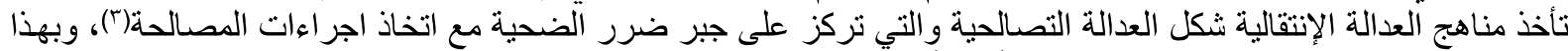

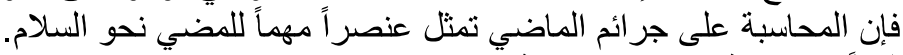

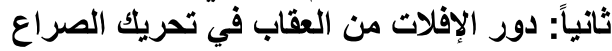

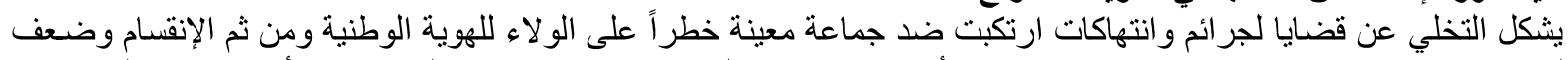

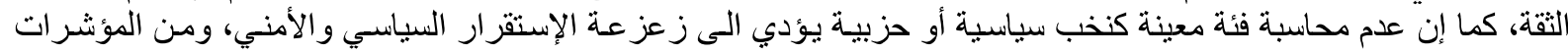

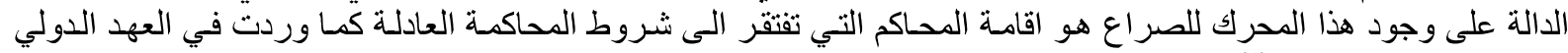

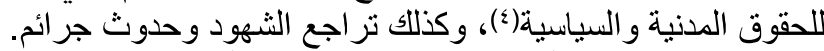

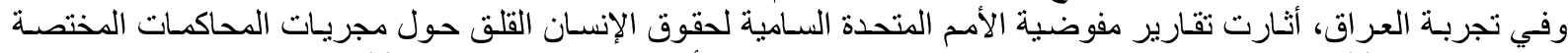

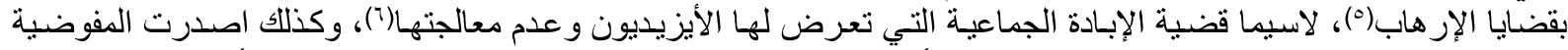

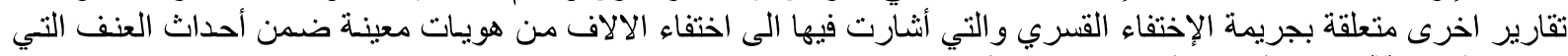
مر بها العراق(V) و لا تزانة ال هذه القضية غير معالجة.

\section{مبدأ جبر الضرر الثاني}

أولاً: جبر الضرر والسلام

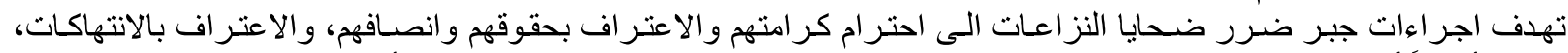

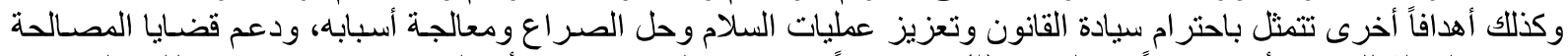

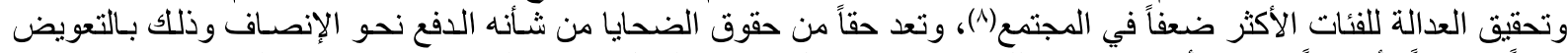

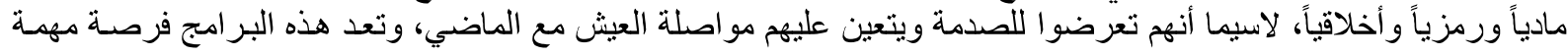

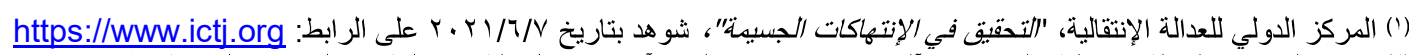

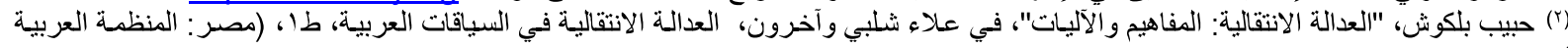

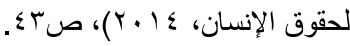
(") ليزا شيرك، استراتيجيات بناء السلام: هل يمكن بناء السلام، ترجمة: هايدي جمال ووجدي وهبة، (مصر: جمعية الأمل العر اقية ودار الثقافة، (1) ب)،

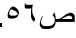

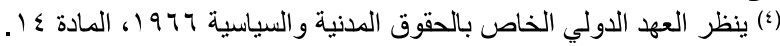

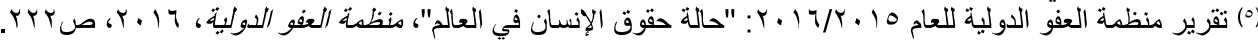

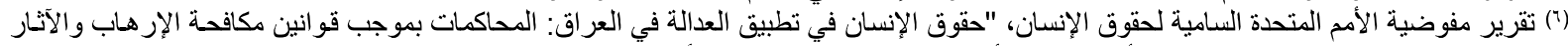

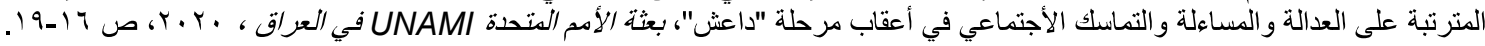
(7) Committee on Enforced Disappearances of United Nation, "Observations on the additional information submitted by Iraq under article 29 (4) of the Convention", CED/C/IRQ/OAI/1, 25/11/2020.

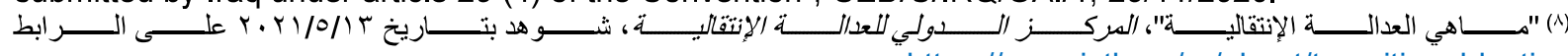
.https://www.ictj.org/ar/about/transitional-justice 
يمكن إستغلالها لقتح قنوات الحوار وتحويل العلاقات العدائية إلى ودية('). وبهذا فلا يمكن تخيل حلول السـلام مع تجاهل كرامـة

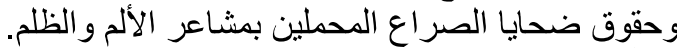

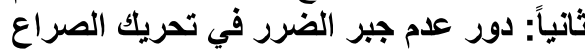

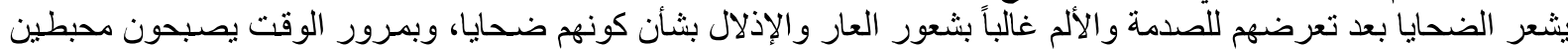

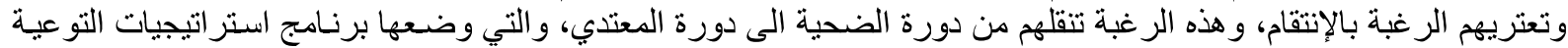

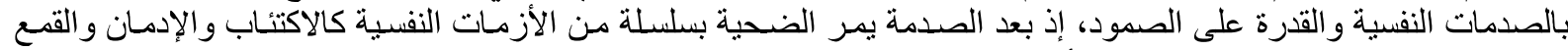

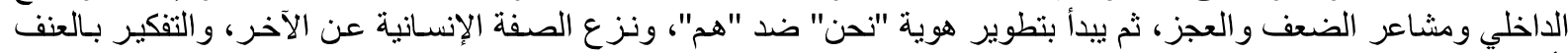

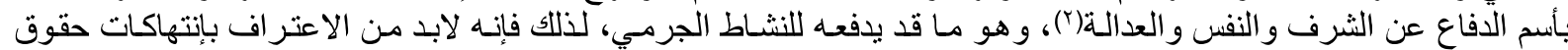

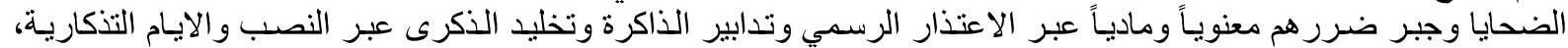

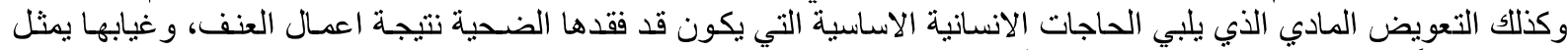
كذللك محركاً للصر الك كما اسلفنا في الفرع الأولاع.

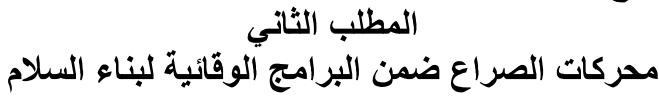

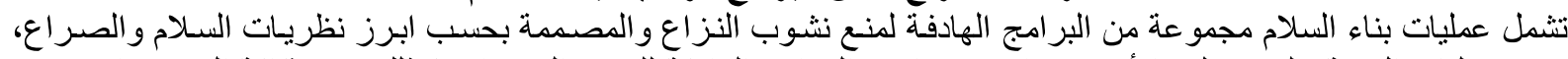

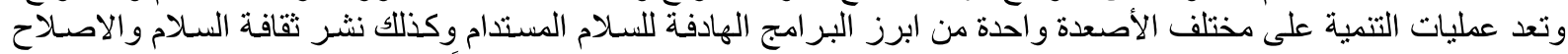

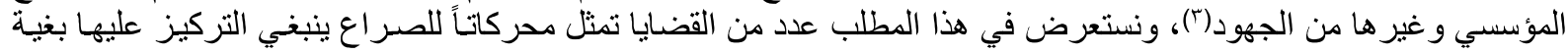

\section{القرع الأول المباول

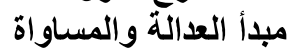

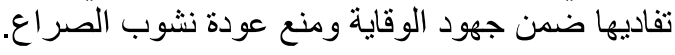

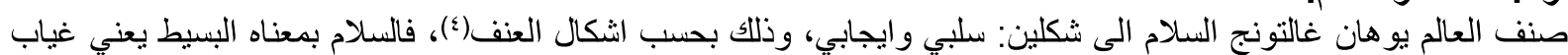

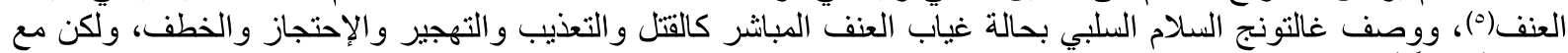

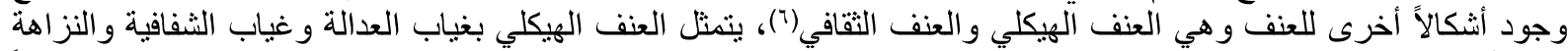

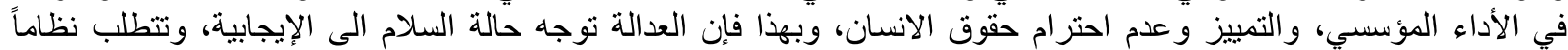

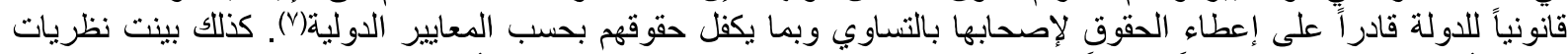

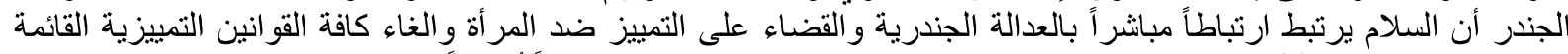

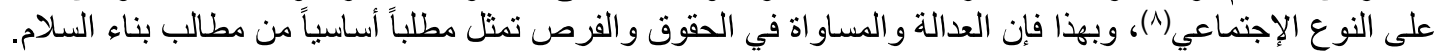

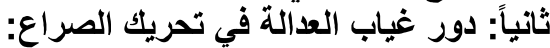

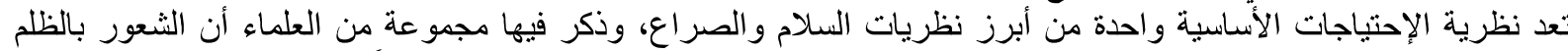

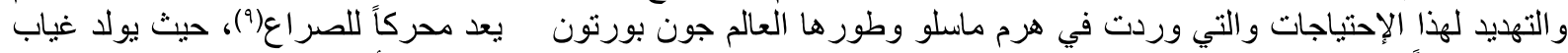

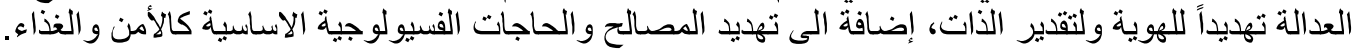

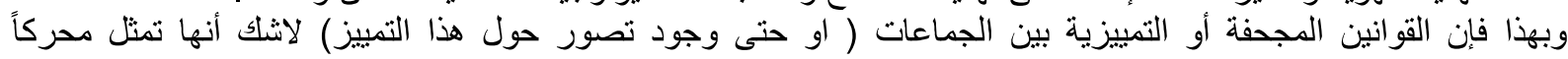

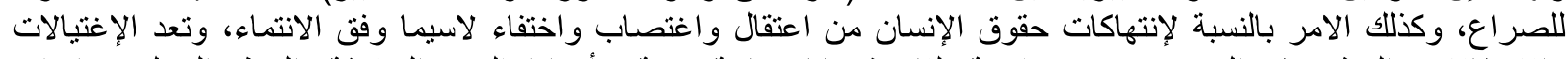

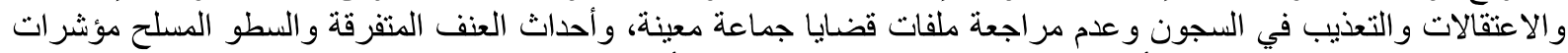

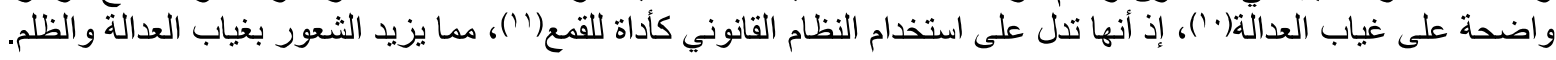

(1) Ruben Carranza, Cristian Correa, and Elena Naughton, "Forms of Justice: A Guide to Designing Reparations Application Forms and Registration Processes for Victims of Human Rights Violations", (USA: International Center of Transitional Justice, 2017), p.4.

() ليسا شيرك، تقبيم الصراع والتخطيط لبناء السلام: نحو نهج تشاركي للأمن الإنساني، ترجمة : حسن ناظم وآخرون، (العراق: جمعية الأمل العر اقية،

(3) Christopher E. Miller, Mary E. King (editor), A glossary of terms and concepts in peace and conflict

studies, $2^{\text {nd }}$ Ed., (USA: University for peace, 2005), pp. 55-56.

(4) Johan Galtung, "An editorial", International Peace Research Institute, journal of peace research, Vol.1, No.1, (1964), pp.1-4.

(5) Angus Stevenson \& Christine A. Lindberg (editor), Oxford Advanced American Dictionary, $3^{\text {rd }}$ Ed., (UK: Oxford University Press, 2010), p.3110.

(6) Johan Galtung, "Violence, Peace, and Peace Research", International Peace Research Institute, journal of peace research, Vol. 6, No. 3, (1969), p. 163.

(7) Andrew Blum," Improving Peacebuilding Evaluation", US Institute of Peace, 2011, p.2.

(8) Christine Chinkin, Mary Kaldor \& Punam Yadav, "Gender and new wars", Stability and International Journal of Security and Development, Vol.9, No.1, (2020), p.1

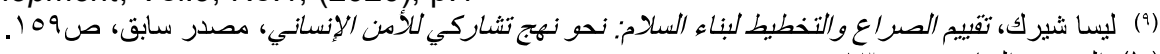

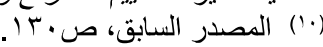

(11) John Agolgia \& others, Measuring Progress in the Conflict Environments: A Metrics Framework, (USA: United States Institutes of Peace, 2010), p.35. 


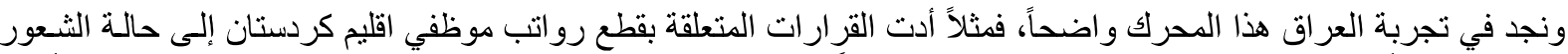

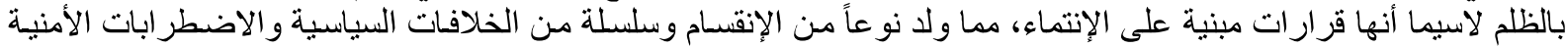

$$
\text { مبدأ شرعية مؤسسآت الدولة }
$$

بين الإقليم و العاصمة العر اقية.

أولاً: شرعية مؤسسات الدولة والسلام:

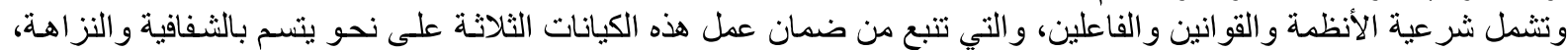

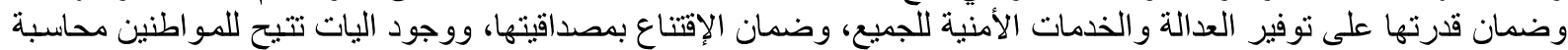

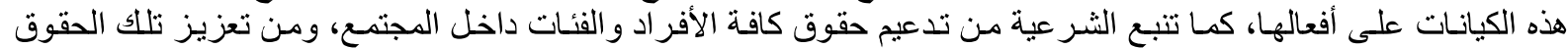

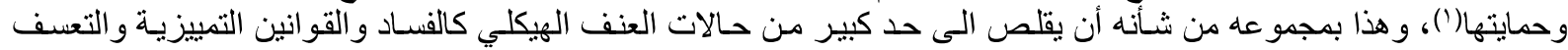

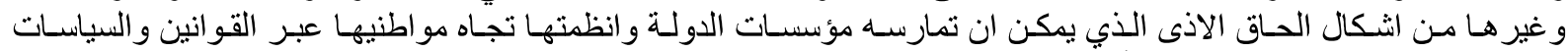

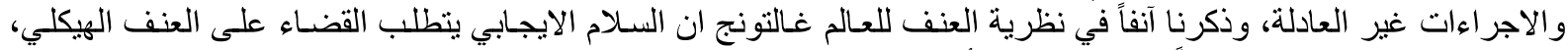

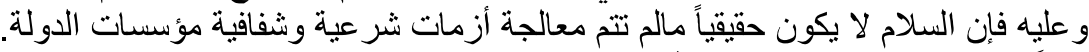

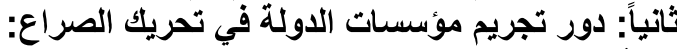

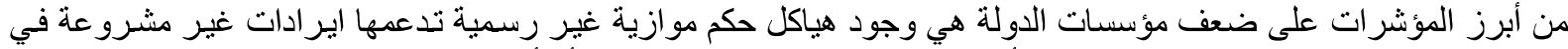

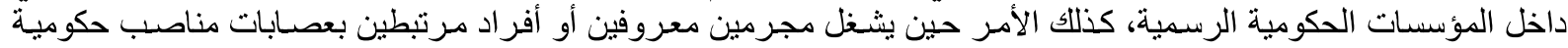

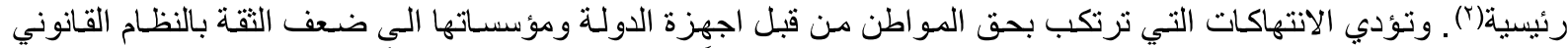

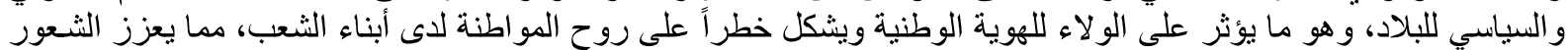

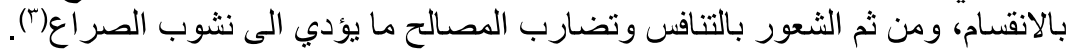
المبحث الثاني

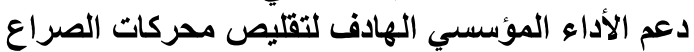

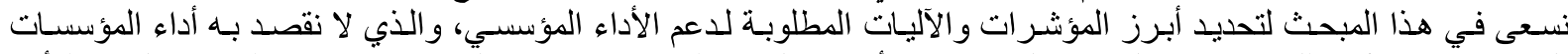

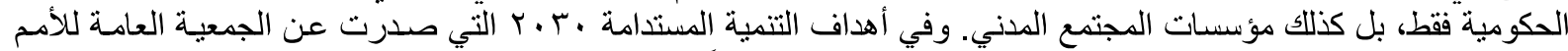

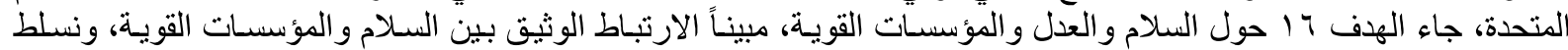

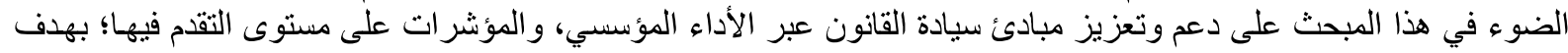

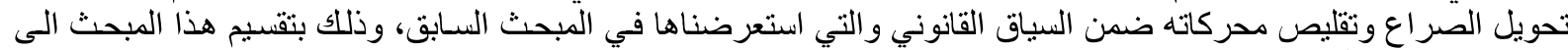

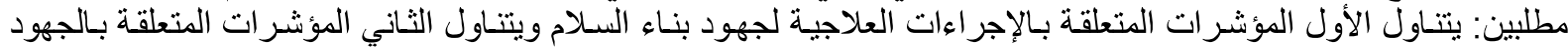

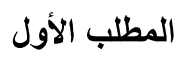

الوقائية له.

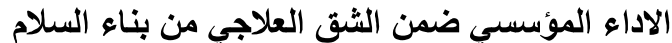

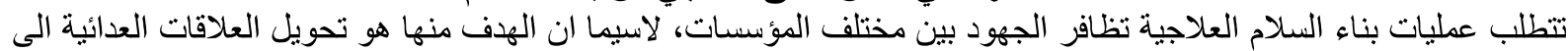

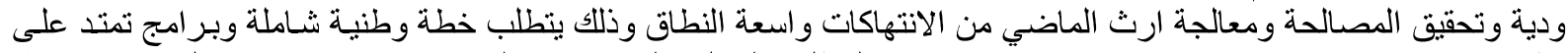

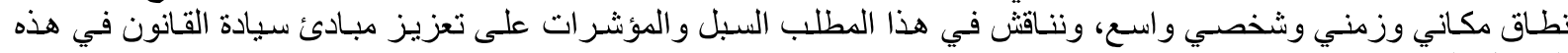

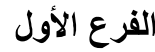

الأمن والنظام العام

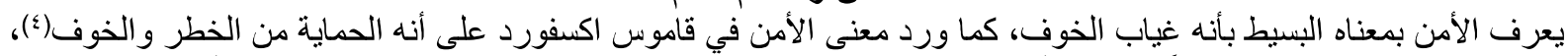

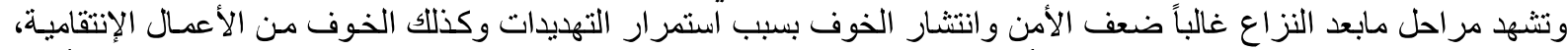

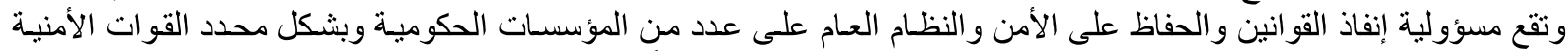

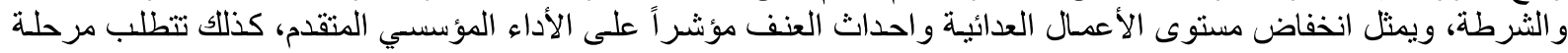

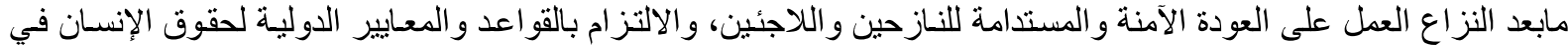

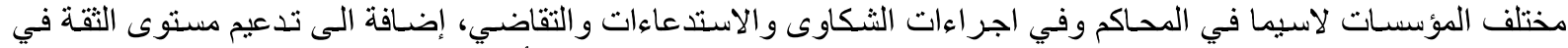
مؤسسـات فرض القانون، ويعد انخفاض معدلات الجريمة والعنف، والحريـة في الأمـاكن العامـة وتزايد معدلات الإبـلاغ عن

(1) ليان مكاي، نحو ثقافة سيادة القانون: استكثـاف الاستجابات الفعالة للتحديات القائمة امام تطبيق العدالة والأمن، (الولايـات المتحدة الأمريكية: معهد

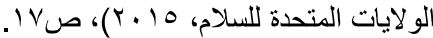
(2)John Agolgia \& others, Measuring Progress in the Conflict Environments: A Metrics Framework, op. cit., p.37.

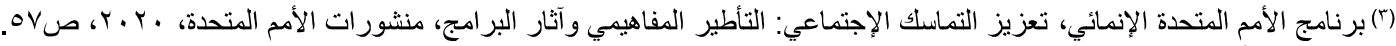

(4) Angus Stevenson \& Christine A. Lindberg (editors), Oxford Advanced American Dictionary, 3rd Ed., (UK: Oxford University Press, 2010). p.3772. 
المخـاطر من أبرز المؤشرات على تزايد مستوى الثقة في أداء المؤسسـات الأمنيـة والمسؤولة عن الحفـاظ على الأمـن النظـام

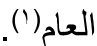

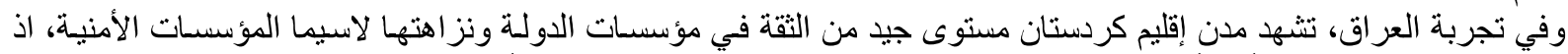

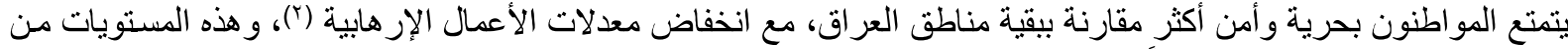
الثقة والحرية قد تكون بذور ها سبياً في تقليص محركات الصر التهاع في الإقليم.

نظام العدالةً الثرأي

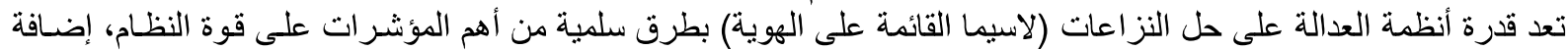

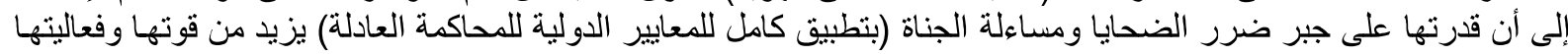

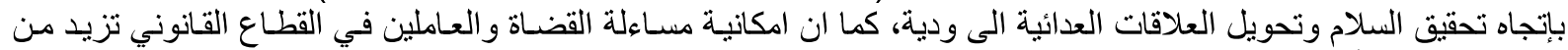

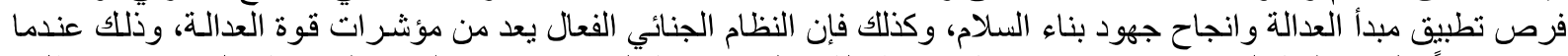

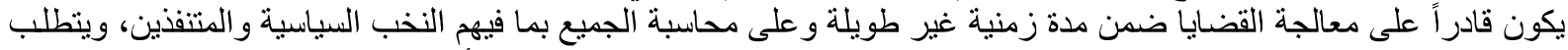

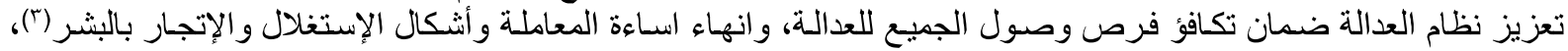

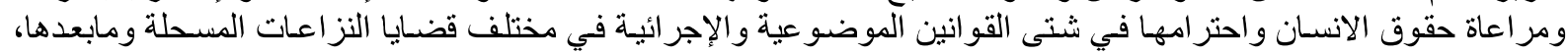

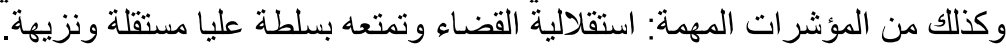

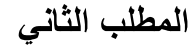

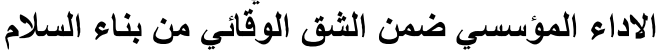

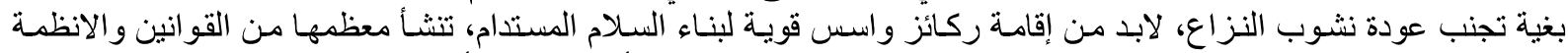

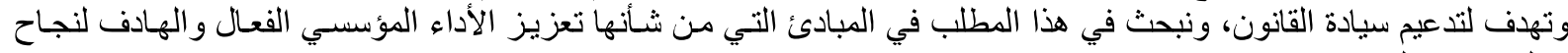

\section{القرع الأول احترام حقوق الإنسان}

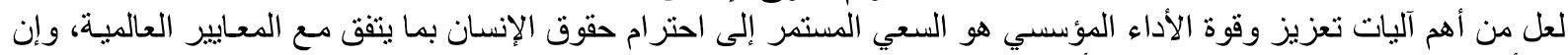

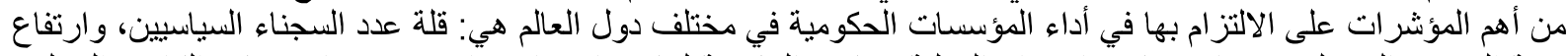

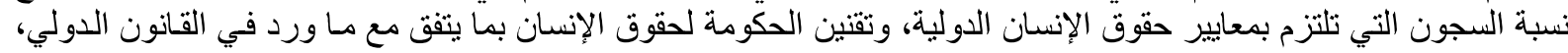

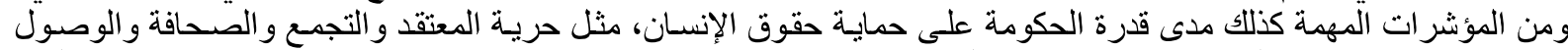

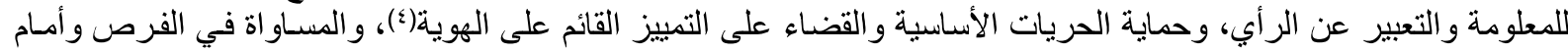
القانون.

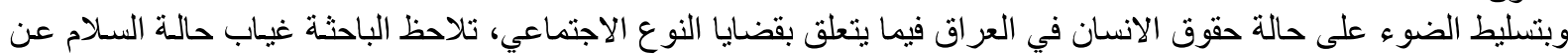

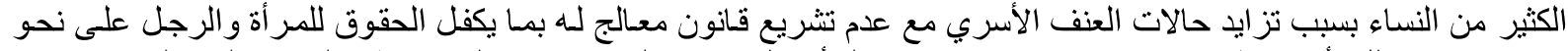

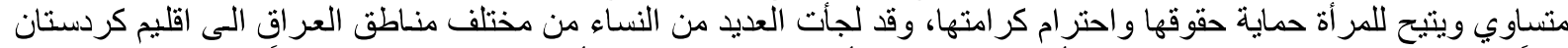

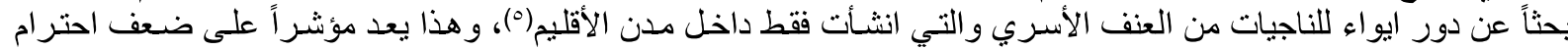

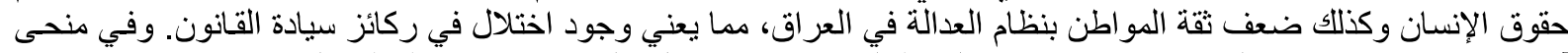

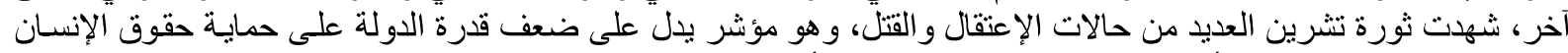

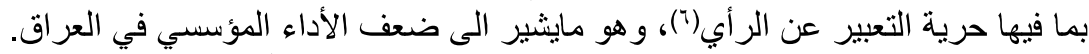

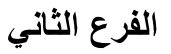

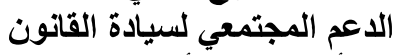

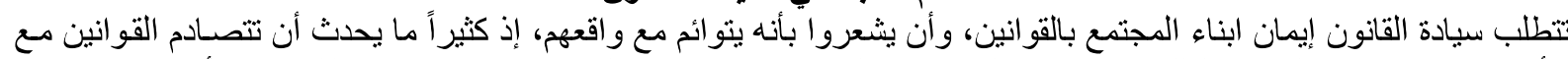

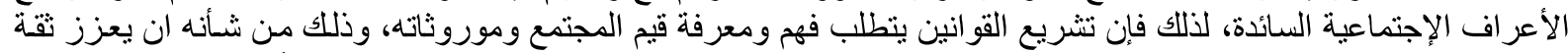

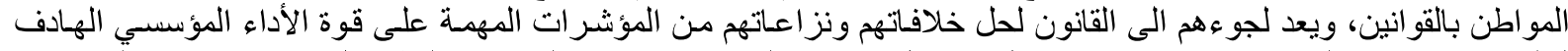

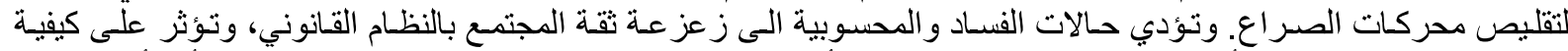

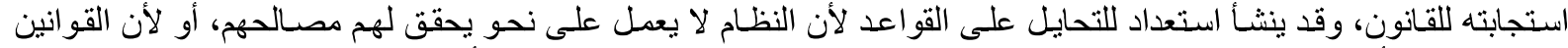

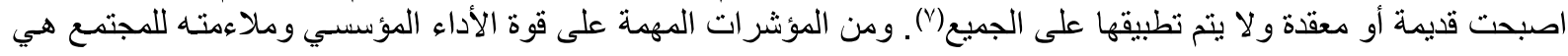

(1) John Agolgia \& others, Measuring Progress in the Conflict Environments: A Metrics Framework, op. cit., p.38-39.

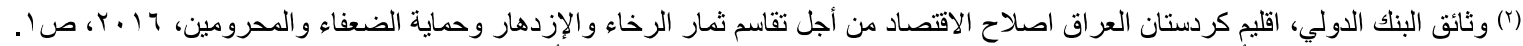

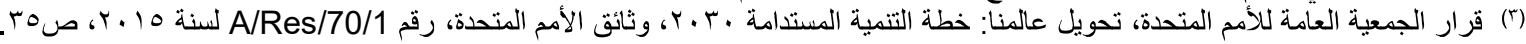

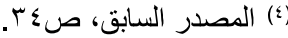

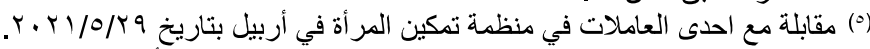

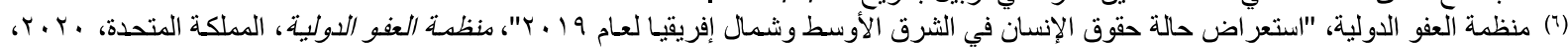




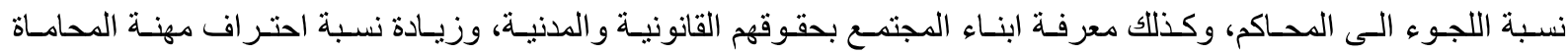
واستقلاليتها.

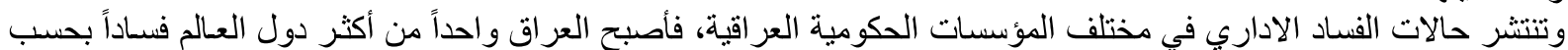

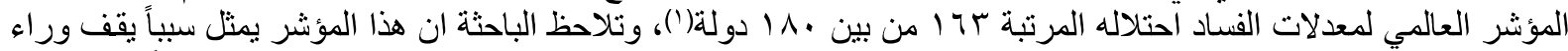

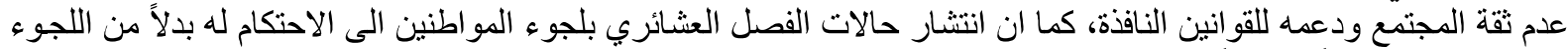

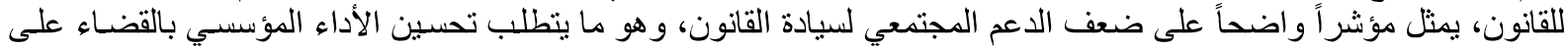

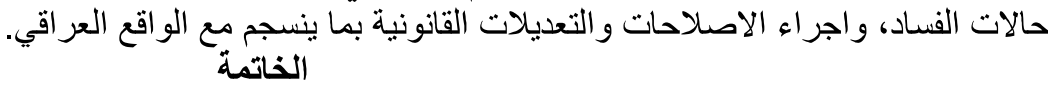

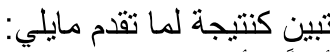

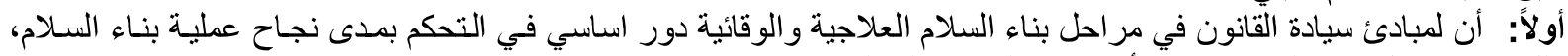

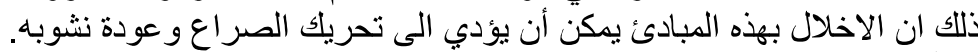

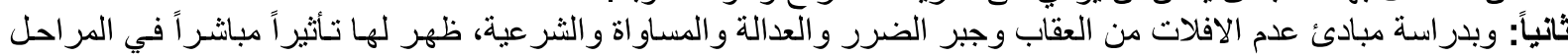

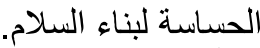

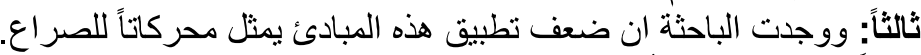

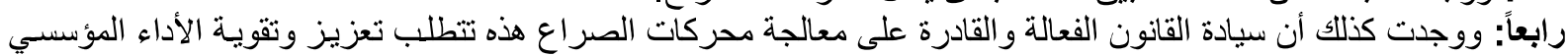

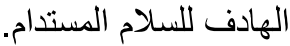

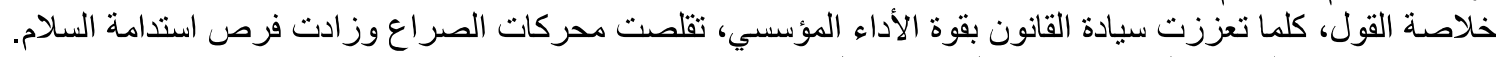

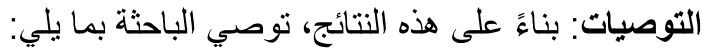

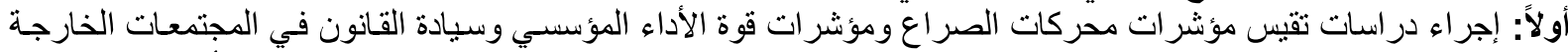

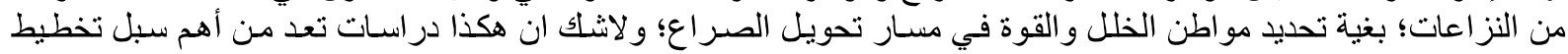

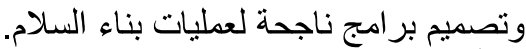

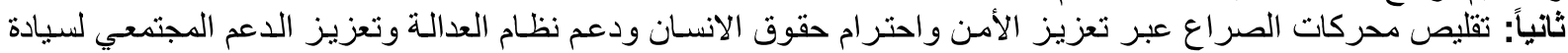

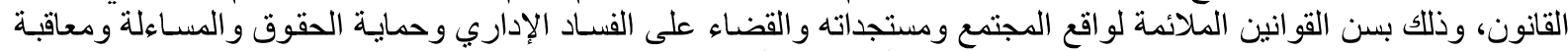

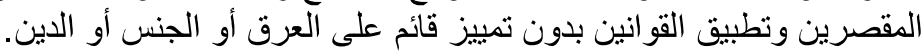

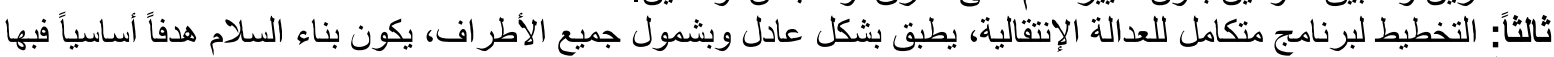

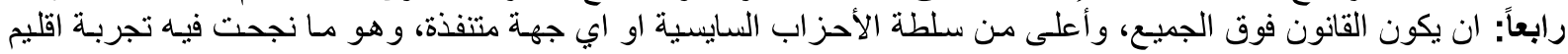

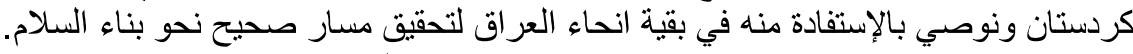
المصادر

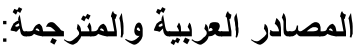

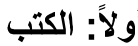
ا. ـ برنامج الأمم المتحدة الإنمائي، تعزيز التماسك الإجتمـاعي: التأطير المفاهيمي وآثار البرامج، (منشورات الأمم المتحدة، . (r.t.

r. . حبيب بلكوش، "العدالة الانتقالية: الدفاهيم والآليات"، في علاء شلبي وآخرون، العدالة الانتقالية في السياقات العربية، طا،

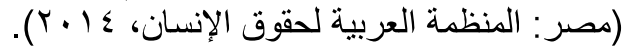

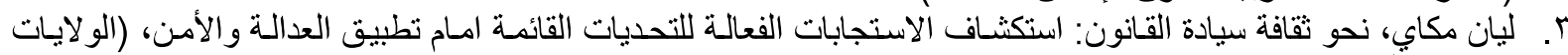

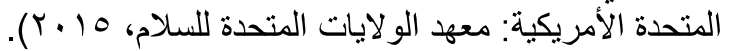

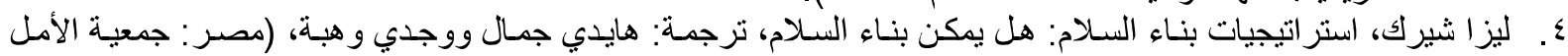

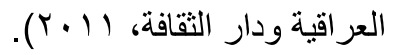

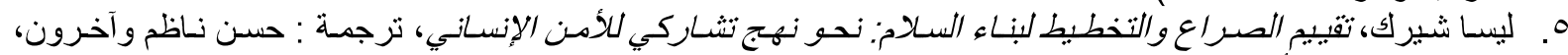

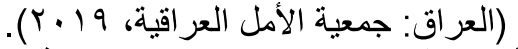

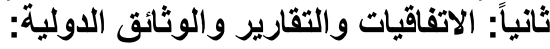

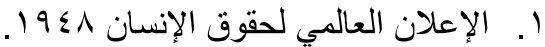

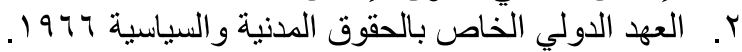

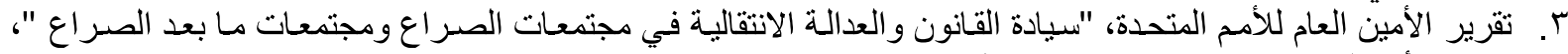

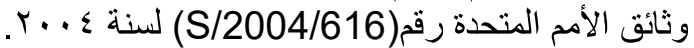

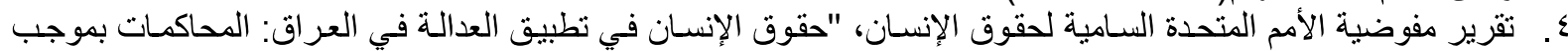

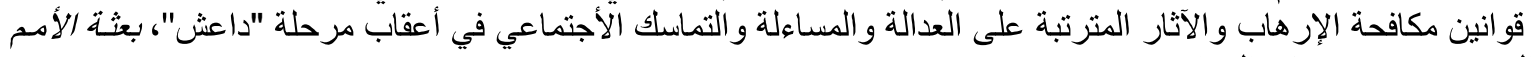

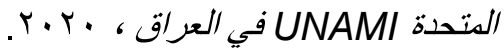

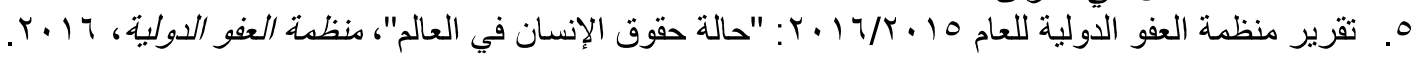

(1) Transparency International, "Corruption Perceptions Index 2019", Germany Transparency International, 2020, p.3. 


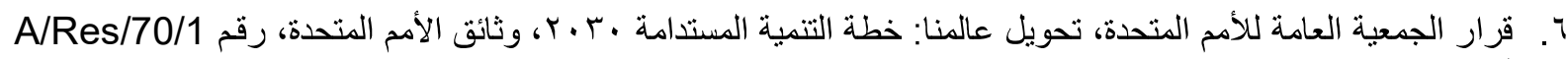
V. و وثائق البنك الدولي، اقليم كردستان العراق اصـلاح الاقتصـاد من أجل تقاسم ثــار الرخـاء والإزدهـار وحمايـة الضـعفاء

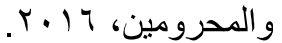
^. . منظمة العفو الدولية، "استعر اض حالة حقوق الإنسان في الثرق الأوسط وشمال إفريقيا لعام 9 ـ ـ ب"، منظمة العفو الدولية،

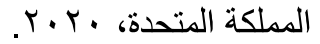

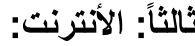

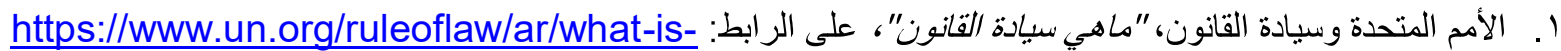
the-rule-of-law

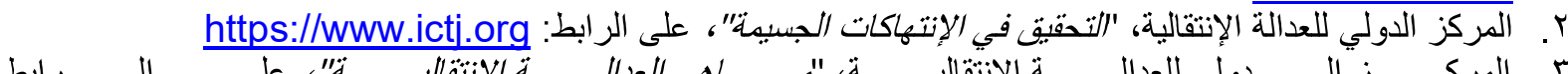

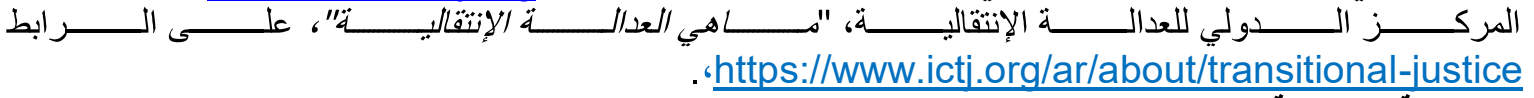

المصادر باللغة الانكليزية

1. Andrew Blum," Improving Peacebuilding Evaluation", US Institute of Peace, 2011.

2. Angus Stevenson \& Christine A. Lindberg (editor), Oxford Advanced American Dictionary, $3^{\text {rd }}$ Ed., (UK: Oxford University Press, 2010).

3. Christine Chinkin, Mary Kaldor \& Punam Yadav, "Gender and new wars", Stability and International Journal of Security and Development, Vol.9, No.1, (2020).

4. Christopher E. Miller, Mary E. King (editor), A glossary of terms and concepts in peace and conflict studies, $2^{\text {nd }}$ Ed., (USA: University for peace, 2005).

5. Committee on Enforced Disappearances of United Nation, "Observations on the additional information submitted by Iraq under article 29 (4) of the Convention", CED/C/IRQ/OAI/1, 25/11/2020.

6. Johan Galtung, "An editorial", International Peace Research Institute, journal of peace research, Vol.1, No.1, (1964).

7. Johan Galtung, "Violence, Peace, and Peace Research", International Peace Research Institute, journal of peace research, Vol. 6, No. 3, (1969).

8. John Agolgia \& others, Measuring Progress in the Conflict Environments: A Metrics Framework, (USA: United States Institutes of Peace, 2010).

9. Ruben Carranza, Cristian Correa, and Elena Naughton, "Forms of Justice: A Guide to Designing Reparations Application Forms and Registration Processes for Victims of Human Rights Violations", (USA: International Center of Transitional Justice, 2017).

10. Transparency International, "Corruption Perceptions Index 2019", Germany Transparency International, 2020.

\section{الملخص}

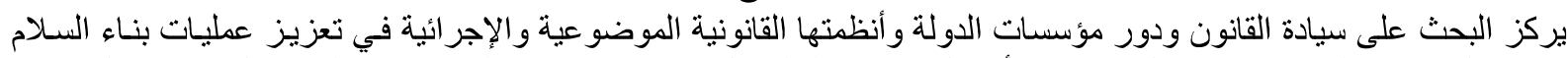

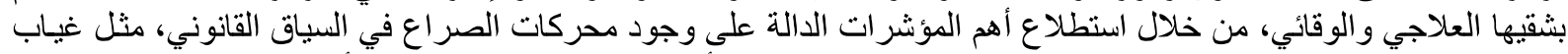

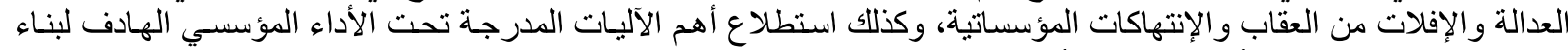

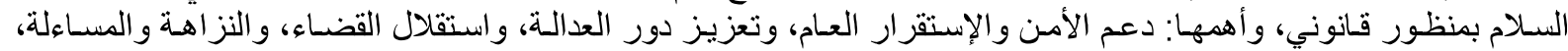

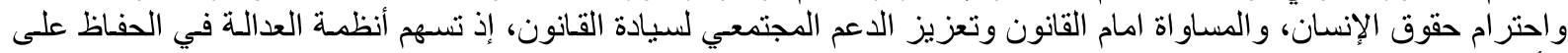

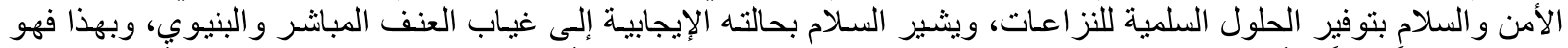

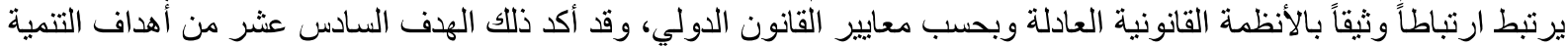

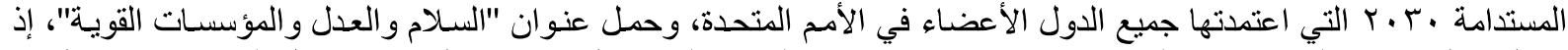

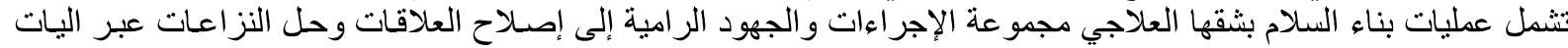

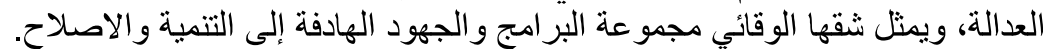

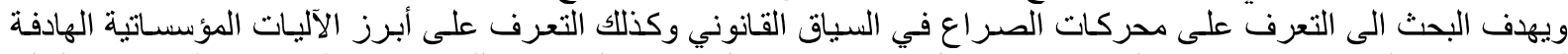

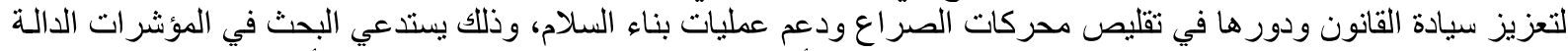

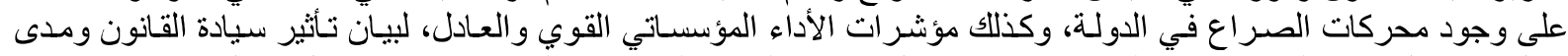

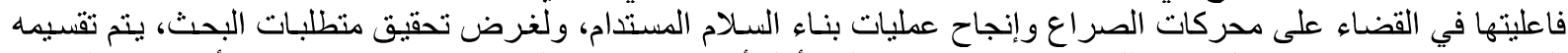

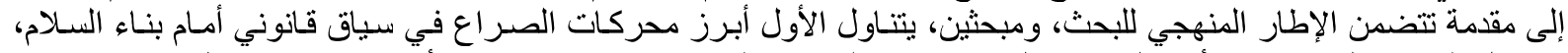

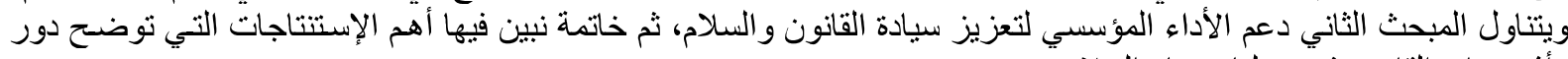
و أثر سيادة القانون في عمليات بناء السعلام. 


$$
\text { الكلمات المفتاحية: بناء السلام، سيادة القانون، محركات الصراع، الأداء المؤسسي. }
$$

\section{Abstract}

The research focuses on the rule of law, the role of state institutions and their substantive and procedural legal systems in promoting peacebuilding processes, both curative and preventive. That by exploring the most important indicators of conflict drivers in the legal context, such as the absence of justice, impunity and institutional violations, as well as exploring the most important mechanisms listed under Institutional performance aimed at building peace with a legal perspective. The most important of which are: supporting security and general stability, strengthening the role of justice, independence of the judiciary, integrity and accountability, respect for human rights, equality before the law and strengthening societal support for the rule of law, as justice systems contribute to maintaining security and peace by providing peaceful solutions. Peace in its positive state refers to the absence of direct and structural violence, and thus it is closely linked to just legal systems and according to international law standards. This was confirmed by the sixteenth goal of the 2030 Sustainable Development Goals adopted by all Member States of the United Nations, titled "Peace, Justice and strong institutions". As peacebuilding operations in their curative part include a set of actions and efforts Aiming at reforming relations and resolving disputes through justice mechanisms, its preventive aspect represents a set of programs and efforts aimed at development and reform.

The research aims to identify the drivers of conflict in the legal context, as well as to identify the most prominent institutional mechanisms aimed at strengthening the rule of law, their role in reducing conflict drivers and supporting peace-building processes. This calls for researching indicators of the presence of conflict drivers in the state, as well as indicators of strong and fair institutional performance. To demonstrate the impact of the rule of law and its effectiveness in eliminating conflict drivers and making sustainable peacebuilding processes successful. For the purpose of achieving the requirements of the research, it is divided into an introduction that includes the methodological framework for research, and two chapters. The first deals with the most prominent drivers of conflict in a legal context in front of peacebuilding, and the second section deals with supporting institutional performance to strengthen the rule of law and peace, and then a conclusion in which we show the most important conclusions that clarify the role and impact of the rule of law in peacebuilding processes.

Keywords: peace building, conflict drivers, institutional performance, rule of law. 